\title{
Manifolds with nonnegative isotropic curvature
}

\author{
HARISH SEShadRI
}

We prove that if $\left(M^{n}, g\right), n \geq 4$, is a compact, orientable, locally irreducible Riemannian manifold with nonnegative isotropic curvature, then one of the following possibilities hold:

(i) $M$ admits a metric with positive isotropic curvature.

(ii) $(M, g)$ is isometric to a locally symmetric space.

(iii) $(M, g)$ is Kähler and biholomorphic to $\mathbb{C} P^{\frac{n}{2}}$.

(iv) $(M, g)$ is quaternionic-Kähler.

This is implied by the following result:

Let $\left(M^{2 n}, g\right)$ be a compact, locally irreducible Kähler manifold with nonnegative isotropic curvature. Then either $M$ is biholomorphic to $\mathbb{C} P^{n}$ or isometric to a compact Hermitian symmetric space. This answers a question of Micallef and Wang in the affirmative.

The proof is based on the recent work of Brendle and Schoen on the Ricci flow.

\section{Introduction}

A Riemannian manifold $(M, g)$ is said to have nonnegative isotropic curvature (NIC) if

$$
R_{1313}+R_{1414}+R_{2323}+R_{2424}-2 R_{1234} \geq 0
$$

for every orthonormal four-frame $\left\{e_{1}, e_{2}, e_{3}, e_{4}\right\}$.

In the case of strict inequality above, we say that the manifold has positive isotropic curvature (PIC). This notion was introduced by Micallef and Moore [10] where they proved that every compact simply connected manifold with PIC is homeomorphic to a sphere. In this paper, we show that the study of compact manifolds (throughout this paper a "compact manifold" will mean a compact manifold without boundary) with NIC reduces to the study of those with PIC. Our main result is the following theorem:

Theorem 1.1. Let $\left(M^{n}, g\right), n \geq 4$, be a compact, orientable, locally irreducible Riemannian manifold with NIC, then one of the following holds:

(i) $M$ admits a metric with PIC. 
(ii) $(M, g)$ is locally symmetric.

(iii) $(M, g)$ is Kähler and biholomorphic to $\mathbb{C} P^{\frac{n}{2}}$.

(iv) $(M, g)$ is quaternionic-Kähler.

We note that according to a recent work of Brendle [2], compact Einstein manifolds with NIC are locally symmetric. Since quaternionic-Kähler manifolds are Einstein, this result of Brendle implies that case (iv) in Theorem 1.1 is included in case (iii).

A version of Theorem 1.1 was proved in dimension 4 by Micallef and Wang [11].

Theorem 1.1 is based on the classification of Kähler manifolds with NIC. We prove the following result which was conjectured by Micallef and Wang [11].

Theorem 1.2. Let $\left(M^{2 n}, g\right), 2 n=\operatorname{dim}_{\mathbb{R}} M \geq 4$, be a compact, locally irreducible Kähler manifold with NIC. Then either $M$ is biholomorphic to $\mathbb{C} P^{n}$ or isometric to a compact Hermitian symmetric space.

Theorem 1.2 along with the uniqueness (up to scaling) of the KählerEinstein metric on $\mathbb{C} P^{n}$ implies the following:

Corollary 1.1. Let $(M, g), \operatorname{dim}_{\mathbb{R}} M \geq 4$, be a compact, locally irreducible Kähler-Einstein manifold with NIC. Then $(M, g)$ is isometric to a compact Hermitian symmetric space.

\section{Remarks}

(i) It follows immediately from Theorem 1.1 that if $(M, g)$ is a compact, orientable, locally irreducible Riemannian manifold with NIC and the dimension of $M$ is odd (which is precisely the case not treated in [11]), then either $M$ admits a PIC metric or $(M, g)$ is locally symmetric.

(ii) The four cases in Theorem 1.1 are not mutually exclusive. An example of a metric, satisfying cases (i) and (ii) in Theorem 1.1, is one of constant positive sectional curvature. The following remark, the proof of which is in Section 4, states that this is the only metric for which that happens:

If $(M, g)$ is one of the following: A compact locally symmetric space of nonconstant sectional curvature, or a compact Kähler manifold or a positive quaternionic-Kähler manifold, then $M$ does not admit a metric of PIC. 
(iii) In [11], Micallef and Wang describe the structure of reducible manifolds with NIC. Combining their result with Theorem 1.1, one can reduce the study of manifolds with NIC to those with PIC.

(iv) The following result is an easy corollary of the Brendle-Schoen theorems mentioned below. The proof of this remark is at the end of this paper.

Let $(M, g)$ be a compact Riemannian manifold with NIC everywhere and PIC at some point. Then $M$ admits a metric with PIC everywhere.

(v) Results analogous to Theorem 1.1 are known (cf. [1,4,12]) for manifolds with nonnegative curvature operator and manifolds with nonstrictly quarter-pinched sectional curvature. Even though NIC is implied by either of these curvature conditions, Theorem 1.1 does not directly imply the earlier results.

The proofs of Theorems 1.1 and 1.2 are based on the fundamental papers of Brendle and Schoen [3,4]. According to their work, if $g(t)$ is the solution to Ricci flow beginning at a metric $g$ with NIC, then $g(t)$ has NIC for all $t$. Moreover, if $F_{t}$ denotes the set of orthonormal four-frames on which the isotropic curvature vanishes, then for $t>0, F_{t}$ is invariant under parallel translation by the Levi-Civita connection of $g(t)$. From this one quickly sees, using the Berger holonomy theorem, that $g(t)$ is either PIC or $g(t)$ has holonomy in $U(m)$ or $\operatorname{Sp}(m) \operatorname{Sp}(1)$, i.e., $(M, g(t))$ is Kähler or quaternionic-Kähler.

Suppose now that $(M, g(t))$ is Kähler. If $J$ is the almost complex structure, then one knows that $F_{t}$ contains all orthonormal frames of the form $\{e, J(e), f, J(f)\}$, where $e$ and $f$ are real tangent vectors. If these are the only elements of $F_{t}$, then a version of Frankel's conjecture due to W. Seaman implies that $M$ is biholomorphic to $\mathbb{C} P^{n}$. On the other hand, if $F_{t}$ has other elements, we will show that the holonomy is a proper subgroup of $U(n)$. Then Berger's theorem will imply that $(M, g(t))$ is symmetric.

Finally, by taking a sequence $t_{i} \rightarrow 0$, we can draw the same conclusions (biholomorphic to $\mathbb{C} P^{n}$ or locally symmetric or quaternionic-Kähler) about $g=\lim _{i \rightarrow \infty} g\left(t_{i}\right)$.

We remark that the Ricci flow was used to study NIC and PIC in $[8,11]$.

\section{NIC and the Ricci flow}

In this section, we recall the results of Brendle and Schoen. Let $(M, h)$ be a compact manifold with NIC. Let $g(t), t \in[0, \varepsilon)$ be the solution to the Ricci 
flow equation

For $t \in(0, \varepsilon)$, let

$$
\frac{\partial g}{\partial t}=-2 \text { Ric }, \quad g(0)=h
$$

$$
\mathcal{F}_{t}=\bigcup_{p \in M}\left\{\left(e_{1}, e_{2}, e_{3}, e_{4}\right) \mid e_{i} \in T_{p} M, \quad g(t)\left(e_{i}, e_{j}\right)=\delta_{i j}, \quad i, j=1, \ldots, 4\right\}
$$

be the bundle of $g(t)$-orthonormal four-frames in $M$. Consider the subset $F_{t} \subset \mathcal{F}_{t}$ defined by

$$
F_{t}:=\left\{\left(e_{1}, e_{2}, e_{3}, e_{4}\right) \mid R_{1313}+R_{1414}+R_{2323}+R_{2424}-2 R_{1234}=0\right\},
$$

where $R$ denotes the curvature tensor of $g(t)$, i.e., $F_{t}$ consists of all $g(t)$ orthonormal four-frames $\left(e_{1}, e_{2}, e_{3}, e_{4}\right)$ at all points of $M$, where the isotropic curvature is zero. The two basic results of Brendle and Schoen that we need are the following: For $t \in(0, \varepsilon)$,

(i) $g(t)$ has NIC (Section 2 in [3]).

(ii) $F_{t}$ is invariant under parallel transport by the Levi-Civita connection of $g(t)$ (Proposition 5 in [4]).

\section{Kähler manifolds with NIC}

This section is devoted to the proof of Theorem 1.2.

Let $(M, g, J), \operatorname{dim}_{\mathbb{R}} M=2 n$, be a compact Kähler manifold with NIC with $J: T M \rightarrow T M$ denoting the almost-complex structure. Let $g(t)$ be the solution to Ricci flow with $g(0)=g$. We fix a $t>0$ and denote $g(t)$ by $g$ and $F_{t}$ by $F$. Note that by choosing $t$ sufficiently small, we can assume that $g(t)$ is locally irreducible. Otherwise, $g=\lim _{t \rightarrow 0} g(t)$ would be locally reducible (this can be seen by considering holonomy groups).

Moreover, since $(M, h)$ is Kähler, so is $(M, g)$. This follows from

Theorem 3.1 (Hamilton [7]). Let $(M, h)$ be a compact Riemannian $n$-manifold. If the restricted holonomy group of $g$ lies in a subgroup of $\mathrm{SO}(n)$, then it continues to do so for $t>0$ under the Ricci flow starting at $h$.

Note that since the restricted holonomy group $\operatorname{Hol}^{0}$ of $(M, g)$ is contained in $U(n)$ by the above theorem, either $(M, g)$ is a Hermitian symmetric space or $\mathrm{Hol}^{0}=U(n)$. In the latter case, $\mathrm{Hol}$ is contained in the normalizer of $\mathrm{Hol}^{0}$ in $\mathrm{SO}(2 n)$ (by the orientability of $M$ ). This normalizer is precisely $U(n)$, and hence $\mathrm{Hol}=U(n)$. In either case, $(M, g)$ is Kähler. 
It can be easily checked that for any Kähler manifold $(M, g, J)$, fourframes of the form $(v, J(v), w, J(w)) \in F$, for any $v, w \in T_{p} M$ satisfying $g(v, w)=g(v, J w)=0$.

The analysis of the Ricci flow splits into two cases, depending on the set $F$.

Case I. $F$ does not contain any frame of the form $(u, J(u), J(v), v)$.

We claim that $(M, g)$ is biholomorphic to $\mathbb{C} P^{n}$ in this case.

For any $p \in M$, let $u, v \in T_{p} M$ with $g(u, v)=g(u, J(v))=0$. As mentioned above, by the symmetries of the curvature tensor of a Kähler manifold, we have $(u, J(u), v, J(v)) \in F$, i.e.,

$$
\begin{aligned}
0= & R(u, v, u, v)+R(u, J(v), u, J(v))+R(J(u), v, J(u), v) \\
& +R(J(u), J(v), J(u), J(v))-2 R(u, J(u), v, J(v)) \\
= & 2(R(u, v, u, v)+R(u, J(v), u, J(v)-R(u, J(u), v, J(v))) .
\end{aligned}
$$

Since $(u, J(u), J(v), v) \notin F$, we have

$$
\begin{aligned}
0< & R(u, J(v), u, J(v))+R(u, v, u, v)+R(J(u), J(v), J(u), J(v)) \\
& +R(J(u), v, J(u), v)-2 R(u, J(u), J(v), v) \\
= & 2(R(u, v, u, v)+R(u, J(v), u, J(v)+R(u, J(u), v, J(v))) .
\end{aligned}
$$

Adding (3.1) and (3.2), we get

$$
R(u, v, u, v)+R(u, J(v), u, J(v))>0
$$

for every $p \in M$ and $u, v \in T_{p} M$ with $g(u, v)=g(u, J(v))=0$. This condition, sometimes referred to as orthogonal bisectional curvature in the literature, is precisely condition $(* *)$ in $[16$, p. 846].

We now state a version of the Frankel conjecture, following $[11,14]$ :

If $\left(M^{2 n}, g\right)$ is a compact locally irreducible Kähler manifold with NIC satisfying (3.3), then $M$ is biholomorphic to $\mathbb{C} P^{n}$.

Proof. The proof is the same as in the paper of Seaman [14] except for some minor changes. For the sake of completeness, we outline it by emphasizing the parts where the curvature assumptions are used. Note that the sign convention for the curvature tensor in $[11,14]$ is the opposite of what we follow. Seaman's version of the Frankel conjecture, Theorem B of [14], asserts the following:

If $\left(M^{2 n}, g\right)$ is a compact Kähler manifold with NIC satisfying (3.4), then $M$ is biholomorphic to $\mathbb{C} P^{n}$ 
where (3.4) is the condition that

$$
R_{i k i k}+R_{i l i l}+R_{j k j k}+R_{j l j l}>0
$$

for all orthonormal vectors $e_{i}, e_{j}, e_{k}$ and $e_{l}$. Our main observation, which is justified in the steps below, is that we can replace (3.4) by (3.3) and local irreducibility of the metric to obtain the same conclusion.

Seaman's proof closely follows the proof of the Frankel conjecture for positive bisectional curvature by Siu and Yau [15]. There are three points where the curvature assumptions play a role:

(i) To start the proof one needs to know that $\pi_{2}(M)=\mathbb{Z} \oplus$ torsion. This is done by showing that $b_{2}(M)=1$ and $\pi_{1}(M)=\{0\}$ and applying the Hurewicz theorem. A reading of the proof by Seaman shows that (3.4) is used to prove that $b_{2}(M)=1$. In our case, we do not have (3.4), instead we have local irreducibility of the metric. This will suffice since Theorem 2.1(b) of Micallef-Wang [11] (note that $b_{2}(M) \neq 0$, since $M$ is Kähler) implies the following: Let $\left(M^{2 n}, g\right)$ be a compact, locally irreducible Riemannian manifold with NIC and $b_{2}(M) \neq 0$. Then $b_{2}(M)=1$ and $M$ is simply connected.

As shown below the only curvature assumptions needed in steps (ii) and (iii) are NIC and positive orthogonal bisectional curvature (3.3).

(ii) Choose a generator $a$ of $H^{2}(M, \mathbb{Z})=\mathbb{Z}$, which is a negative multiple of the Kähler class, and $\alpha$ be a generator of the free part of $\pi_{2}(M)$ such that $a(\alpha)=1$. By a result of Kobayashi-Ochiai [9], to show that $M$ is biholomorphic to $\mathbb{C} P^{n}$, it is enough to show that $c_{1}(M)(\alpha) \geq n+1$, where $c_{1}(M)$ denotes the first Chern class of $M$. By the Sacks-Uhlenbeck theorem [15, Theorem 5.5], we may represent the free homotopy class of $\alpha$ by $\sum_{i=1}^{k} f_{i}$, where each $f_{i}$ is harmonic and energy minimizing in its free homotopy class. One claims that each $f_{i}$ is \pm holomorphic. To see this, one needs the complex formulation of isotropic curvature and the second variation formula for the energy of minimal surfaces as in the paper of Micallef-Moore [10]. We recall this below and prove the holomorphicity lemma in detail, following Seaman:

Let $(M, g)$ be a Riemannian manifold. Let $E=T M \otimes \mathbb{C}$. Extend $g$ to a symmetric bilinear form $($,$) and a Hermitian form \langle\langle\rangle$,$\rangle on E$. For $p \in M$, an element $v \in E_{p}$ is said to be isotropic if $(v, v)=0$. A two-plane $P \subset E_{p}$ is isotropic, if every element of $P$ is isotropic. It can be checked that $\{v, w\}$ spans an isotropic two-plane if and only if $v$ and $w$ are linearly independent and

$$
(v, v)=(w, w)=(v, w)=0
$$


Also, $\operatorname{Span}_{\mathbb{C}}\{v, w\}$ is an isotropic two-plane if and only if there exist orthonormal vectors $e_{1}, e_{2}, e_{3}$ and $e_{4}$ in $T_{p} M$ such that

$$
v=\frac{\|v\|}{\sqrt{2}}\left(e_{1}+\sqrt{-1} e_{2}\right), \quad w=\frac{\|w\|}{\sqrt{2}}\left(e_{3}+\sqrt{-1} e_{4}\right),
$$

where the norm is with respect to $\langle\langle\rangle$,$\rangle .$

Let

$$
\mathcal{R}: \bigwedge^{2} E_{p} \rightarrow \bigwedge^{2} E_{p}
$$

denote the complex linear extension of the curvature operator. If $v, w$, $e_{1}, \ldots, e_{4}$ are as above, then it can be checked that

$$
4\langle\langle\mathcal{R}(v \wedge w), v \wedge w\rangle\rangle=\|v\|^{2}\|w\|^{2} R^{i s o}\left(e_{1}, e_{2}, e_{3}, e_{4}\right),
$$

where for any tangent vectors $X, Y, Z, W$

$$
\begin{aligned}
R^{\text {iso }}(X, Y, Z, W):= & R(X, Z, X, Z)+R(X, W, X, W)+R(Y, Z, Y, Z) \\
& +R(Y, W, Y, W)-2 R(X, Y, Z, W) .
\end{aligned}
$$

The assumption in case $\mathrm{I}$ is that

$$
R^{\text {iso }}(X, J(X), J(Y), Y)>0
$$

for any orthonormal frame $(X, J(X), J(Y), Y)$.

Let $f: S^{2} \rightarrow M$ be a smooth immersion. Consider $F=f^{*}(E)=f^{*} T M \otimes$ $\mathbb{C}$. We pull-back $g,($,$) and \langle\langle\rangle$,$\rangle and denote them by the same symbols. F$ also carries the pull-back of the Levi-Civita connection on $M$, extended complex linearly. This connection is Hermitian with respect to $\langle\langle\rangle$,$\rangle . It is well$ known that a Hermitian bundle $V$ on a Riemann surface $\Sigma$ with a Hermitian connection $\nabla$ can be endowed with a holomorphic structure $\bar{\partial}$, in which a section $s$ of $V$ is holomorphic if and only if

$$
\nabla_{\frac{\partial}{\partial \bar{z}}} s=0
$$

in any local holomorphic coordinate $z$ on $\Sigma$.

Consider $S^{2}$ as $\mathbb{C} \cup\{\infty\}$ and let $Z$ be the holomorphic vector field, which is $\frac{\partial}{\partial z}$ on $\mathbb{C}$ and 0 at $\infty$. If $f$ is a harmonic map, then $f_{*} Z$ is a holomorphic isotropic section of $F$, where $f_{*}: T S^{2} \otimes \mathbb{C} \rightarrow F$. If, in addition, $f$ is stable and $(M, g)$ has NIC, then it follows from the stability inequality of Micallef 
and Moore that

$$
\left\langle\left\langle\mathcal{R}\left(s \wedge f_{*} Z\right), s \wedge f_{*} Z\right\rangle\right\rangle=0
$$

for any holomorphic section $s$ of $F$ such that $s$ and $f_{*} Z$ span an isotropic two-plane.

Now we can prove that a harmonic map into a Kähler manifold with NIC and positive orthogonal bisectional curvature has to be \pm holomorphic, i.e., $J \circ f_{*}(Z)= \pm \sqrt{-1} f_{*}(Z)$.

Proof. Let $S=\left\{x \in S^{2} \mid\left(f_{*} Z\right)_{x}=0\right\}$. Then $S$ is a finite set. Take $p \in S^{2} \backslash S$.

Let $w=\left(f_{*} Z\right)_{p}$ and $F_{p}=f^{*} T_{p} M \otimes \mathbb{C}$ as before. We can write $F_{p}=$ $F_{p}^{(1,0)} \oplus F_{p}^{(0,1)}$, where $F_{p}^{(1,0)}$, and $F_{p}^{(0,1)}$ are the eigenspaces of $J$ for the eigenvalues $\sqrt{-1}$ and $-\sqrt{-1}$, respectively.

It is enough to prove that $w$ is an eigenvector of $J$. Suppose not. Now $w$ and $J(w)$ span an isotropic two-plane. Also, we can write $w=w^{\prime}+w^{\prime \prime}$ as the sum of nonzero $(1,0)$ and $(0,1)$ parts. Since $w$ is also isotropic, we can find orthonormal $e_{1}, e_{2} \in f^{*} T_{p} M$ such that $g\left(e_{1}, J\left(e_{2}\right)\right)=0$ and

$$
\sqrt{2} \frac{w^{\prime}}{\left\|w^{\prime}\right\|}=e_{1}-\sqrt{-1} J\left(e_{1}\right), \quad \sqrt{2} \frac{w^{\prime \prime}}{\left\|w^{\prime \prime}\right\|}=e_{2}+\sqrt{-1} J\left(e_{2}\right) .
$$

Since $w \wedge J(w)=-2 \sqrt{-1} w^{\prime} \wedge w^{\prime \prime},(3.5)$ gives

$$
\begin{aligned}
& \langle\langle\mathcal{R}(w \wedge J(w)), w \wedge J(w)\rangle\rangle=4\left\langle\left\langle\mathcal{R}\left(w^{\prime} \wedge w^{\prime \prime}\right), w^{\prime} \wedge w^{\prime \prime}\right\rangle\right\rangle \\
& =\left\|w^{\prime}\right\|^{2}\left\|w^{\prime \prime}\right\|^{2}\left(R^{\text {iso }}\left(e_{1},-J\left(e_{1}\right), e_{2}, J\left(e_{2}\right)\right)\right) \\
& =\left\|w^{\prime}\right\|^{2}\left\|w^{\prime \prime}\right\|^{2}\left(R^{\text {iso }}\left(e_{1}, J\left(e_{1}\right), J\left(e_{2}\right), e_{2}\right)\right)>0,
\end{aligned}
$$

which contradicts (3.6). Hence $w$ is an eigenvector for $J$ on the complement of finitely many points in $S^{2}$, i.e., $f$ is \pm holomorphic on this set. It follows that $f$ is \pm holomorphic on all of $S^{2}$.

(iii) By (ii), we have

$$
\alpha=\sum_{i=1}^{k} f_{i},
$$

where each $f_{i}$ is \pm holomorphic. In [16, pp. 853-854], Seaman proves the following fact:

Let $\left(M^{n}, g\right)$ be a compact Kähler manifold with positive orthogonal bisectional curvature and let $T M$ denotes the holomorphic tangent bundle of $M$. Let $f: S^{2} \rightarrow M$ be any nonconstant holomorphic map. By a theorem of 
Grothendieck, $f^{*} T M=L_{1} \oplus \cdots \oplus L_{n}$, where the $L_{i}$ 's are holomorphic line bundles. The claim is that

$$
c_{1}\left(L_{i}\right) \geq 1 \forall i \quad \text { and } \quad c_{1}\left(L_{i_{0}}\right) \geq 2 \text { for some } i_{0}
$$

We refer the readers to [14] for a proof.

This result implies that if $k=1$ in (3.7) and $f_{1}$ is holomorphic, then $c_{1}(M)(\alpha)=c_{1}(M)\left[f_{1}^{*}\left(S^{2}\right)\right]=c_{1}\left(f_{1}^{*}(T M)\right)\left[S^{2}\right] \geq n+1 . \quad$ Actually, $\quad k=1$ implies that $f_{1}$ is holomorphic, since $c_{1}(M)$ is a negative multiple of the Kähler class. In order to show that $k=1$, one proves the deformation lemma [17, Proposition 3, p. 201]. Given this Lemma the proof that $k=1$ is identical to that of Claim 2 of [17, pp. 202-203].

As was observed by Futaki [5], the deformation lemma holds for any compact Kähler manifold and any holomorphic map $f: S^{2} \rightarrow M$ as long as $f^{*} T M$ splits as a direct sum of positive line bundles. See [5] or the last part in $[16$, p. 854] for a proof.

Hence we conclude that $M$ is biholomorphic to $\mathbb{C} P^{n}$ under the assumptions of Case I.

Case II. $F$ contains a frame of the form $(u, J(u), J(v), v)$.

We claim that the restricted holonomy group of $(M, g)$ cannot be the whole group $U(n) \subset \mathrm{SO}(2 n)$. This will complete the proof of Theorem 1.2 by Berger's holonomy theorem.

Suppose $\operatorname{Hol}^{0}(M)=U(n)$.

The fact that $(u, J(u), v, J(v))$ and $(u, J(u), J(v), v)$ both belong to $F$ gives (as in (3.1) and (3.2))

$$
\begin{aligned}
R(u, J(u), v, J(v))= & R(u, J(v), u, J(v))+R(u, v, u, v) \\
& +R(J(u), J(v), J(u), J(v))+R(J(u), v, J(u), v)=0
\end{aligned}
$$

which, by the symmetries of Kähler curvature, gives

$$
R(u, J(u), v, J(v))=R(u, J(v), u, J(v))+R(u, v, u, v)=0 .
$$

We now use the holonomy action of $U(n)$ repeatedly to get a contradiction. First, there is an element of $U(n)$ under which

$$
u \rightarrow \frac{1}{\sqrt{2}}(u-J(v)), \quad v \rightarrow \frac{1}{\sqrt{2}}(u+J(v)) .
$$

Hence $\left(\frac{1}{\sqrt{2}}(u-J(v)), \frac{1}{\sqrt{2}}(J(u)+v), \frac{1}{\sqrt{2}}(u+J(v)), \frac{1}{\sqrt{2}}(J(u)-v)\right) \in F$. 
The equation corresponding to (3.8) is

$R\left(\frac{1}{\sqrt{2}}(u-J(v)), \frac{1}{\sqrt{2}}(J(u)+v), \frac{1}{\sqrt{2}}(u+J(v)), \frac{1}{\sqrt{2}}(J(u)-v)\right)=0$.

This equation gives

$$
\begin{aligned}
R(u & -J(v), J(u)+v, u+J(v), J(u)-v) \\
= & R(u, J(u), u, J(u))+R(u, J(u), u,-v)+R(u, J(u), J(v), J(u)) \\
& +R(u, J(u), J(v),-v)+R(u, v, u, J(u))+R(u, v, u,-v) \\
& +R(u, v, J(v), J(u))+R(u, v, J(v),-v) \\
& +R(-J(v), J(u), u, J(u))+R(-J(v), J(u), u,-v) \\
& +R(-J(v), J(u), J(v), J(u))+R(-J(v), J(u), J(v),-v) \\
& +R(-J(v), v, u, J(u))+R(-J(v), v, u,-v) \\
& +R(-J(v), v, J(v), J(u))+R(-J(v), v, J(v),-v)=0 .
\end{aligned}
$$

The sum of the second, third, fifth and ninth terms on the right side is

$$
\begin{aligned}
& R(u, J(u), u,-v)+R(u, J(u), J(v), J(u))+R(u, v, u, J(u)) \\
& \quad+R(-J(v), J(u), u, J(u))=0 .
\end{aligned}
$$

Similarly, the sum of the 8 th, 12 th, 14 th and 15 th terms is

$$
\begin{aligned}
& R(u, v, J(v),-v)+R(-J(v), J(u), J(v),-v)+R(-J(v), v, u,-v) \\
& \quad+R(-J(v), v, J(v), J(u))=0
\end{aligned}
$$

The sum of the 6 th, 7 th, 10 th and 11 th terms is

$$
\begin{aligned}
& R(u, v, u,-v)+R(u, v, J(v), J(u))+R(-J(v), J(u), u,-v) \\
& \quad+R(-J(v), J(u), J(v), J(u))=-4 R(u, v, u, v) .
\end{aligned}
$$

The sum of the 4 th and 13 th terms is

$$
R(u, J(u), J(v),-v)+R(-J(v), v, u, J(u))=0
$$

by (3.8). 
Using these four equations and simplifying the expansion of $R(u-J(v)$, $J(u)+v, u+J(v), J(u)-v)$, we get

$$
R(u, J(u), u, J(u))+R(v, J(v), v, J(v))-4 R(u, v, u, v)=0 .
$$

Next, consider the element of $U(n)$ which takes

$$
u \rightarrow \frac{1}{\sqrt{2}}(u-v), \quad v \rightarrow \frac{1}{\sqrt{2}}(u+v) .
$$

Now the equation corresponding to (3.10) (obtained by substituting $-J(v)$ for $v$ in $(3.10))$ is

$$
R(u, J(u), u, J(u))+R(v, J(v), v, J(v))-4 R(u, J(v), u, J(v))=0 .
$$

Combining (3.12), (3.10) and (3.8) gives

$$
\begin{aligned}
& R(u, v, u, v)=R(u, J(v), u, J(v)) \\
& \quad=R(u, J(u), u, J(u))+R(v, J(v), v, J(v))=0 .
\end{aligned}
$$

Extend $\left\{e_{1}=u, e_{2}=v\right\}$ to an orthonormal basis $\left\{e_{1}, J\left(e_{1}\right), e_{2}, J\left(e_{2}\right), \ldots\right.$, $\left.e_{n}, J\left(e_{n}\right)\right\}$. By considering the element of $U(n)$ which interchanges $u$ and $e_{i}, J(u)$ and $J\left(e_{i}\right)(i \geq 3)$ and keep the other elements of the basis fixed, we see that $\left(e_{i}, J\left(e_{i}\right), J(v), v\right) \in F$. A similar operation on $v$ shows that $\left(e_{i}, J\left(e_{i}\right), e_{j}, J\left(e_{j}\right)\right) \in F$, for all $1 \leq i \leq n, 1 \leq j \leq n, i \neq j$. The equation corresponding to $(3.13)$ is

$$
\begin{aligned}
& R\left(e_{i}, e_{j}, e_{i}, e_{j}\right)=R\left(e_{i}, J\left(e_{j}\right), e_{i}, J\left(e_{j}\right)\right) \\
& \quad=R\left(e_{i}, J\left(e_{i}\right), e_{i}, J\left(e_{i}\right)\right)+R\left(e_{j}, J\left(e_{j}\right), e_{j}, J\left(e_{j}\right)\right)=0 .
\end{aligned}
$$

The equation $R\left(e_{i}, J\left(e_{i}\right), e_{i}, J\left(e_{i}\right)\right)+R\left(e_{j}, J\left(e_{j}\right), e_{j}, J\left(e_{j}\right)\right)=0$, for all $i \neq j$, clearly implies that $R\left(e_{i}, J\left(e_{i}\right), e_{i}, J\left(e_{i}\right)\right)=0$ for all $i$. In particular $R(u, J(u), u, J(u))=0$. If $w$ is an arbitrary unit vector, then we can find an element $T$ in $U(n)$ taking $u$ to $w$. By considering the frame $(w, J(w), J(T(v))$, $T(v)) \in F$, we get $R(w, J(w), w, J(w))=0$. Hence the holomorphic sectional curvature of $g$, is zero, which implies that $g$ is flat and hence locally reducible.

This contradiction completes the proof of Theorem 1.2.

\section{The classification theorem}

We now outline the proof of Theorem 3.2. Let $(M, g)$ be a compact, orientable, locally irreducible manifold of NIC. Let $g(t), t \in[0, \delta)$ denotes the 
solution to Ricci flow with $g(0)=g$. By [3], $g(t)$ has NIC. Suppose $g(t)$ does not have strictly PIC for any $t \in(0, \delta)$. Then, $F_{t} \neq \phi$ for all $t \in(0, \delta)$. This implies that $\operatorname{Hol}^{0}(M, g(t)) \neq \mathrm{SO}(n)$ for all such $t$. Suppose $\operatorname{Hol}^{0}\left(M, g\left(t_{0}\right)\right)=$ $\mathrm{SO}(n)$ for some $t_{0}$. This assumption along with the invariance of $F_{t_{0}}$ under parallel transport implies that every orthonormal four-frame at every point would be in $F_{t_{0}}$, i.e., every isotropic curvature at every point would be zero. As scalar curvature can be expressed as the sum of isotropic curvatures (see Proposition 4.1 below), this implies that $R=0$ for $\left(M, g\left(t_{0}\right)\right)$. Since the initial metric $g$ has nonnegative scalar curvature, by the maximum principle for scalar curvature along the flow, the scalar curvature of $(M, g(t))$ is identically zero for all $t \in\left[0, t_{0}\right]$. From the evolution equation for $R$ under Ricci flow,

$$
\frac{\partial R}{\partial t}=\triangle R+|R i c|^{2}
$$

it follows that $\operatorname{Ric}(g)=0$.

Moreover, we have the following:

Proposition 4.1 (Proposition 2.5 of [11]). A metric g with positive (resp. nonnegative) isotropic curvature has positive (resp. nonnegative) scalar curvature. If the scalar curvature is identically zero, then $g$ must be conformally flat.

Therefore, $g$ is also conformally flat and hence flat, contradicting local irreducibility.

Hence, we conclude that $\operatorname{Hol}^{0}(M, g(t))$ is a proper subgroup of $\mathrm{SO}(n)$ for all $t \in(0, \delta)$. As before, for sufficiently small $t$, say $t<\delta_{1}, g(t)$ will also be locally irreducible.

Take any $t^{\prime} \in\left(0, \delta_{1}\right)$. If $\left(M, g\left(t^{\prime}\right)\right)$ is not locally symmetric, then $\mathrm{Hol}^{0}$ would have to be either $U(m)$ or $\operatorname{Sp}(m) \operatorname{Sp}(1)$, with $m$ equal to $n / 2$ or $n / 4$, respectively. The other possibilities for $\mathrm{Hol}^{0}$ in the Berger holonomy theorem can be ruled out, since they would again lead to scalar flatness or local symmetry.

In case $\mathrm{Hol}^{0}=U(m)$, the full holonomy group $\mathrm{Hol}=U(m)$ as well, since $\mathrm{Hol}$ is contained in the normalizer of $\mathrm{Hol}^{0}$ in $\mathrm{SO}(n)$ (note that we are assuming the orientability of $M$ here). This normalizer is precisely $U(n)$. Since Hol $=U(m),\left(M, g\left(t^{\prime}\right)\right)$ is Kähler and Theorem 1.2 implies either $(M, g)$ is biholomorphic to $\mathbb{C} P^{n}$ or a symmetric space.

If $\mathrm{Hol}^{0}=\mathrm{Sp}(m) \mathrm{Sp}(1)$, then again $\mathrm{Hol}=\mathrm{Sp}(m) \mathrm{Sp}(1)$ (note that since $\mathrm{Sp}(m) \mathrm{Sp}(1)$ is a maximal subgroup of $\mathrm{SO}(4 n)[6]$, its normalizer in $\mathrm{SO}(4 n)$ is itself). Hence for any $t^{\prime} \in\left(0, \delta_{1}\right)$ either $\left(M, g\left(t^{\prime}\right)\right)$ is Kähler and biholomorphic to $\mathbb{C} P^{n}$ or a locally symmetric space or quaternionic-Kähler. By 
taking a sequence $t_{i} \rightarrow 0$ as $i \rightarrow \infty$ and noting that $g\left(t_{i}\right) \rightarrow g$ in $C^{\infty}$, we can conclude the same about $(M, g)$. This completes the proof of Theorem 1.1.

Proof of Remark (ii). First, by the results of [11] or [14], if $(M, h)$ has PIC, then $H^{2}(M, \mathbb{R})=\{0\}$. Hence $M$ cannot admit a Kähler metric. If $M$ admitted a positive quaternionic-Kähler metric, then it would be simply connected and $H^{4}(M, \mathbb{R}) \neq\{0\}$. But since $M$ admits a PIC metric, it would be homeomorphic to a sphere, a contradiction.

Suppose that there is a locally symmetric metric $g$ on $M$. Note that $M$ admits a metric of positive scalar curvature (namely $h),(M, g)$ will have to be a locally symmetric space of compact type. In particular, $(M, g)$ is Einstein with positive scalar curvature. By the Bonnet-Myers theorem, $M$ has finite fundamental group and hence the universal cover $\tilde{M}$ is a compact manifold. The Micallef-Moore theorem applied to $(\tilde{M}, \tilde{h})$ implies that $\tilde{M}$ is homeomorphic to a sphere. For topological reasons, the only locally symmetric metric on a topological sphere is one of constant positive sectional curvature. Therefore, $\tilde{g}$ (and $g$ ) would have to be of constant positive sectional curvature.

Proof of Remark (iv). Suppose $K^{\text {iso }} \geq 0$ on $M$ and $K^{\text {iso }}(p)>0$ for some $p \in M$. With notation as earlier, if $F_{t}$ is not empty, i.e., if $g(t)$ does not have $K^{\text {iso }}>0$, then by the invariance under parallel transport of $F_{t}$, there is a four-frame for which $K^{\text {iso }}=0$ at every point of $M$. In particular, there is such a frame at $p$. Hence we have a time-dependent four-frame $\left(e_{1}(t), e_{2}(t)\right.$, $\left.e_{3}(t), e_{4}(t)\right)$ at $p$ for which $K^{\text {iso }}=0$. We can choose a sequence of times $t_{i} \rightarrow 0$ as $i \rightarrow \infty$ for which the corresponding sequence of frames converges to an orthonormal frame on $(M, g)$. This frame will satisfy $K^{\text {iso }}=0$ contradicting $K^{\text {iso }}>0$ at $p$.

\section{Acknowledgments}

The author is very grateful to Simon Brendle for pointing out errors in the original draft and to Claude LeBrun for helpful suggestions. This work was supported by DST Grant No. SR/S4/MS-283/05.

\section{References}

[1] C. Böhm, and B. Wilking, Manifolds with positive curvature operators are space forms, Ann. of Math. 167(3) (2008), 1079-1097. 
[2] S. Brendle, Einstein manifolds with nonnegative isotropic curvature are locally symmetric, Duke Math J. 151(1) (2010), 1-21.

[3] S. Brendle and R. Schoen, Manifolds with 1/4-pinched curvature are space forms, J. Am. Math. Soc. 22 (2009), 287-307.

[4] S. Brendle and R. Schoen, Classification of manifolds with weakly 1/4pinched curvatures, Acta Math. 200 (2008), 1-13.

[5] A. Futaki, On compact Kähler manifolds with semi-positive bisectional curvature, J. Fac. Sci. Univ. Tokyo 1A, Math. 28 (1981), 111-125.

[6] A. Gray, A note on manifolds whose holonomy group is a subgroup of $S p(n) S p(1)$, Mich. Math. J. 16 (1965), 125-128.

[7] R.S. Hamilton, The formation of singularities in the Ricci flow, Surveys in differential geometry, II (Cambridge, MA, 1993), Int. Press, Cambridge, MA, 1995, 7-136.

[8] R.S. Hamilton, Four-manifolds with positive isotropic curvature, Comm. Anal. Geom. 5(1) (1997), 1-92.

[9] S. Kobayashi and T. Ochiai, Characterization of complex projective spaces and hyperquadrics, J. Math. Kyoto Univ. 13 (1973), 31-47.

[10] M. Micallef and J. Moore, Minimal two-spheres and the topology of manifolds with positive curvature on totally isotropic two-planes, Ann. of Math. (2) 127(1) (1988), 199-227.

[11] M. Micallef and M. Wang, Metrics with nonnegative isotropic curvature, Duke Math. J. 72(3) (1993), 649-672.

[12] L. Nei and B. Wu, Complete manifolds with nonnegative curvature operator, Proc. AMS 135 (2007), 3021-3028.

[13] J. Sacks and K. Uhlenbeck, The existence of minimal immersions of 2-spheres, Ann. of Math. 113 (1981), 1-24.

[14] W. Seaman, On manifolds with nonnegative curvature on totally isotropic 2-planes, Trans. Am. Math. Soc. 338(2) (1993), 843-855.

[15] Y.-T. Siu and S.-T. Yau, Compact Kähler manifolds with positive bisectional curvature, Invent. Math. 59 (1980), 189-204. 
Department of MATHEMAtics

Indian Institute of ScIEnCE

BANGALORE 560012

INDIA

E-mail address: harish@math.iisc.ernet.in

Received December 8, 2008 
